\title{
Chronobiology and meal times: internal and external factors
}

\author{
BY JIM WATERHOUSE ${ }^{1}$, D. MINORS ${ }^{2}$, G. ATKINSON ${ }^{1}$ AND D. BENTON $^{3}$ \\ ${ }^{1}$ School of Human Sciences, Liverpool John Moores University, Liverpool \\ ${ }^{2}$ School of Biological Sciences, University of Manchester, Manchester \\ ${ }^{3}$ Department of Psychology, University of Wales Swansea, Swansea
}

\begin{abstract}
Although homeostatic mechanisms remain of utmost importance, rhythmic changes are present also. The main ones have a period of $24 \mathrm{~h}$ (circadian) or about $2-3 \mathrm{~h}$ (ultradian). Circadian rhythms are derived from a body clock, found in the base of the brain, and from the pattern of our sleepwake cycle, including activity and meal times. These rhythms promote the regular changes between an active wake period and a recuperative sleep period. Ultradian rhythms are also widespread and reflect external (lifestyle) and internal factors. The internal factors include biochemical need and some sort of oscillator; but details of how many oscillators, and exactly where they are, remain to be established. Food intake, appetite, digestion and metabolism have been shown to illustrate these principles. Moreover, these principles become important when special circumstances exist as far as meal times are concerned; the particular difficulties of night workers is a good example.
\end{abstract}

Eating behaviour: Rhythmicity of food intake

The concept of homeostasis is a key one in physiology and biochemistry; it describes the observations that, for example, core temperature and arterial blood pressure are maintained within a narrow band in spite of changes in the heat load and direction of gravitational forces to which the body is normally exposed, and blood glucose is maintained within narrow limits despite discontinuous intake of food.

Recent technological developments (for examples, see Wever, 1979; Minors \& Waterhouse, 1981; Moore-Ede et al. 1982) have enabled an ever-increasing range of variables to be monitored, often non-invasively, while the subject is living normally (movement, mental performance, brain electrical activity); biochemical developments have enabled very small volumes of blood or saliva to be collected at frequent intervals and assayed for a whole range of substances. Such developments have not called into question the viability of the concept of homeostasis but they have shown that the limits within which a variable is controlled homeostatically show a circadian (of about $24 \mathrm{~h}$ ) period. In addition, ultradian (episodic when applied to hormones) periods exist which can vary from $20 \mathrm{~h}$ to smaller values; in the present paper, we will consider those that show a period of $1-5 \mathrm{~h}$ (for glossary of terms, see Table 1 ). What causes these rhythms, and how much do they influence food intake in non-obese subjects not suffering from food-intake disorders?

\section{CIRCADIAN RHYTHMS}

These are present in almost all physiological and biochemical variables and nearly always show a mean period of exactly $24 \mathrm{~h}$ in subjects living normally (Minors \& Waterhouse, 1981; Moore-Ede et al. 1982). Some of these rhythms can be attributed to our lifestyle. For example, stature increases while asleep (due to changes in intervertebral disc height) and 
Table 1. Glossary

\begin{tabular}{ll}
\hline \hline Circadian & A period of about $24 \mathrm{~h}(20-28 \mathrm{~h})$ \\
Constant routine & An experimental protocol in which exogenous components are removed \\
Endogenous & Due to an internal influence such as the circadian or ultradian oscillator \\
Exogenous & Due to an external influence such as food intake, physical activity, ambient lighting \\
Non-24h 'day' & A protocol by which the subject lives in accord with a timepiece that is running fast (for \\
example, twenty-four apparent hours equal twenty-one solar hours) or slow (for example, \\
twenty-four apparent hours equal twenty-seven solar hours) \\
A period of less than $20 \mathrm{~h}(2-3 \mathrm{~h}$ in the article) \\
An extremal rhythmic influence (for example, the light-dark or eating-fasting cycles) that \\
Zeitgeber
\end{tabular}

falls in the hours following waking and adopting again an upright posture (Reilly, 1990). Such a rhythm would be termed 'exogenous' to indicate its origin. By contrast, most rhythms show, to an extent that is characteristic of the rhythm under consideration, an additional component that appears to derive from some form of internal timing mechanism or 'body clock'. This is called the endogenous component of the rhythm.

Methods have been devised to distinguish between these endogenous and exogenous components of a measured rhythm. They are based on the fact that the body clock (see below) is slow to adjust to a change in lifestyle. Briefly, some of the experimental protocols, and the interpretation of the results they produce, are as follows (see Minors \& Waterhouse, 1981).

\section{Constant routines}

If a subject remains awake in a constant environment, and remains sedentary or supine and takes identical regular meals, the exogenous component is removed. The circadian rhythm that remains is that due to the endogenous component.

\section{Changed sleep-activity schedules}

These can mimic shift work, a time-zone transition or living on a non- $24 \mathrm{~h}$ 'day'. The exogenous component of a rhythm will adjust immediately to the change in lifestyle whereas the body clock does so only slowly (in the case of a time-zone transition), not at all (with a non-24h 'day') or only to a limited extent (shift work).

\section{PROPERTIES OF THE CIRCADIAN CLOCK}

The timekeeping properties of the body clock have been investigated in individuals in a time-free environment (for example, an underground cave or specially sound-proofed room; see Minors \& Waterhouse, 1981). In such circumstances the timing of the individual's lifestyle (the time of waking in particular) can be determined only by an internal clock. To some extent, meal times and retiring will be determined by estimates of the passage of time, as judged by things done, etc., but this would be, at best, rather erratic and influenced by the subject's activities (Aschoff $e t$ al. 1986). Experiments have shown that such individuals live surprisingly regularly but the period of their lifestyle (for example, the amount of time between successive sleep terminations) is closer to $25 \mathrm{~h}$ than $24 \mathrm{~h}$ (Wever, 1979). This observation has given rise to the term circadian (from the Latin 
for 'about a day') but implies that the body clock is continually adjusted to an exact $24 \mathrm{~h}$ day when living in a normal environment. This is achieved by zeitgebers ('time givers') in the environment. These are rhythmic inputs and the main one in human subjects is the light-dark cycle. It is likely that under normal circumstances this is reinforced by other cycles, contenders being some or all rhythmic changes in social influences, mental activity, physical activity and food intake (Czeisler et al. 1981). The pineal hormone, melatonin, is another important influence and will be described later (see below).

If work from rodents is applicable to human subjects (and the species are similar neuroanatomically, even though important differences exist in the factors causing food intake, see below), the body clock is present in the paired hypothalamic suprachiasmatic nuclei (SCN; Moore, 1992). These nuclei receive afferents directly from the eye via a retinohypothalamic tract, which is probably the route by which the light-dark cycle adjusts the body clock, as well as from many other regions of the brain, particularly via the intergeniculate leaflet. There is also a humoral input from the pineal gland, with receptors for melatonin being present on SCN cells (Reppert et al. 1988). Outputs from the SCN pass to hypothalamic nuclei that control temperature regulation and hormone secretion as well as to the raphé nucleus (concerned with sleep) and the sympathetic nervous system. As a result, the clock can exert its effects throughout the body. The output from the sympathetic nervous system is particularly important as it goes to the pineal gland and controls production of melatonin by influencing the rate-limiting enzyme of the anabolic pathway. It is believed that the feedback loop back to the SCN (via melatonin) exerts an important controlling influence on the SCN oscillators (Lewy et al. 1992).

\section{ROLE OF THE CIRCADIAN CLOCK}

The role of the body clock is not only to increase body activity during the daytime but also to decrease it, and so conserve energy and promote restitution, during nocturnal sleep. The clock is important also in allowing the future to be predicted, thus allowing preparations for sleep and waking (Moore-Ede, 1986). It is a common experience, for example, that the evening is a time of decreasing alertness and increasing readiness for sleep itself, and many physiological and biochemical changes accompany such progressions. Human subjects cannot suddenly go to sleep, nor can they suddenly awaken and work efficiently; the clock, often aided by our chosen lifestyle, enables us to prepare for these profound changes in neurophysiology.

Such a clock needs to be 'robust', that is, not to be influenced by transient changes in lifestyle, such as a daytime nap or a nocturnal waking to visit the larder or an infant who needs to be fed. However, in human subjects, in whom lifestyle changes can be marked, this property results in problems. After a time-zone transition or during night work, in both of which cases the timing of individuals' lifestyle is changed in a major way, the normal synchrony between lifestyle and the body clock is temporarily lost. As a consequence the individuals' ability to sleep and perform physical and mental work, as well as their desire to eat, will no longer match their lifestyle; they will suffer from 'jet-lag' or 'shiftworkers' malaise' until the zeitgebers in their new environment cause the body clock to adjust appropriately (Minors \& Waterhouse, 1981; Moore-Ede et al. 1982). Whilst this occurs after a few days following time-zone transitions, it is much slower, and generally incomplete, during night work. This is because all zeitgebers in the new time-zone are timed to promote adjustment, but during nightwork there is a conflict between the zeitgebers, some changing (for example, the sleep-activity cycle) but other not (the natural light-dark cycle and rhythms in society as a whole). 


\section{ULTRADIAN RHYTHMS}

These form a more heterogeneous group than circadian rhythms, differing far more widely than circadian rhythms in their period and, probably, origin (Lavie, 1980; Lavie \& Kripke, 1981). The following two examples will illustrate some of the principles.

Most hormones show a very complex concentration profile when measured over $1 \mathrm{~d}$ or so. One difficulty of interpretation is that the plasma concentration reflects losses by metabolism and elimination as well as secretion. However, it is possible to separate these two processes mathematically (Veldhuis, 1994). The method uses the known half-time constants describing the decay in concentration of the hormone in plasma produced by the processes of distribution and metabolism to estimate the size and frequency of the secretory pulses. It indicates that the size and/or frequency of secretory pulses exhibits an ultradian rhythm. In several hormones, the pulses originate in a 'pulse generator' found in hypothalamic neurosecretory nuclei and believed to result from some form of neuronal feedback loop within a particular nucleus (Maeda et al. 1995). There is an input to these pulse generators from the SCN (see p. S31) which results in a circadian rhythm of pulsatile activity. In practice, therefore, most hormones show a combination of circadian and pulsatile (ultradian) changes, these latter originating in the hypothalamus or elsewhere (for example, the islets of Langerhans; Stagner et al. 1980). Exogenous influences exist also; whether these are produced by posture, food or fluid intake, or environmental factors such as light and stress, depends on the hormone.

A second example of ultradian rhythmicity is that of the cycling between rapid eye movement (REM) sleep and the other, non-REM, stages of sleep (stages 1 and 2, and slowwave sleep or stages 3 and 4); the REM-nonREM cycle. This lasts about $90 \mathrm{~min}$ in adults and starts with the non-REM component after sleep onset. The basic cycle is believed to originate from interactions between several regions of the brain; the raphe system is an important contributor. It has been suggested (Kleitman, 1982) that this cycle continues during our waking lives (where it is manifest as variations in the amount of mental activity and an alternation between left- and right-cerebral hemispheric dominance), but the evidence for this connection is not strong. Several factors influence this REM-nonREM cycle and successive cycles differ in the details of their composition. The initial non-REM components are rich in slow-wave sleep, the amount being determined by previous wake time and the later components are richer in stage 2 sleep. By contrast, the amount of REM sleep in successive cycles generally increases since it is inversely proportional to core temperature which is at a minimum at about 05.00 hours (Dijk \& Czeisler, 1995).

Ultradian rhythms also show the influence of both exogenous and endogenous factors. The role of ultradian rhythms, in particular of the period shown by a variable, is not always clear (but see Lloyd \& Kippert, 1993). For clinical studies involving hormone therapy (for example, for delayed puberty), the evidence is that sustained levels of hormone in the plasma produce down-regulation in the target tissue (Editorial, 1984); pulsatile infusion, by analogy with the natural episodic secretion, is more effective clinically.

In adults, circadian and ultradian oscillations are different outputs from different oscillators. Thus, in mammals, as indicated by surgical removal, the 'body clock' and ultradian 'pulse generator' appear to be anatomically distinct and can function independently of each other.

\section{RHYTHMS OF FOOD INTAKE}

In adults there are circadian and ultradian patterns of food intake and similar rhythms of gut motility, secretion of digestive juices and absorption of digested foods, and of 
concentrations of carbohydrates, amino acids and lipids in the bloodstream (Lavie, 1980; Jorde et al. 1987; Tarquini et al. 1987; Mejean et al. 1988).

When the origins of such patterns are considered, they are clearly related to individuals' habits and to social norms. Do these rhythms, in addition, reflect endogenous oscillatory drives? Studying an individual in social isolation, even in the absence of time cues, does little to resolve the issue. Thus, even if such an individual shows a $30 \mathrm{~h}$ 'day' and continues to take the same number and distribution of meals and snacks as on a normal $24 \mathrm{~h}$ day, this does not indicate unambiguously that an oscillator controlling the sleep-wake cycle also directly controls food intake. This is because meals and snacks are an integral part of our daily routine; when we eat, and often the kind of meal we eat, seems to be determined by conditioned reflexes, routine and by a feeling that 'it is time to eat', rather than biological need (for example, see Aschoff et al. 1986). A similar interpretation can be made of the observation that meal times in individuals living at high latitudes change little throughout the year in spite of wide variations in the number of daylight hours (P. Lappalainen, personal communication). However, even this factor can be overridden; for example, during the holy month of Ramadan (Khashoggi et al. 1993). The further observation (Bernstein et al. 1981) that other subjects living in isolation showed a significant positive correlation between meal size and the interval to the next meal suggests that metabolic factors, in addition to effects of habit and an internal oscillator, also play a role. In summary, no studies seem to have investigated in detail the factors that determine why we choose to eat, our appetite before meals, and the amount of food we eat, at different times of the day.

In babies, such feeding patterns will not already have been laid down; this, coupled with the observation that ultradian rhythms are more marked and circadian rhythms are less marked than in the adult (Hellbrugge, 1973; Honnebier et al. 1989), offers the opportunity to investigate possible origins of ultradian feeding patterns in neonates. Certainly, the act of feeding neonates on demand was associated with rises in their heart rate, blood pressure and general behavioural activity (Weinert et al. 1994). Therefore, does the baby's desire for food reflect the activity of some internal ultradian oscillator, which also influences the cardiovascular system? Further observations of feeding times in these demand-fed babies indicated that a periodicity (of about $4 \mathrm{~h}$ ) existed throughout the $24 \mathrm{~h}$, but this could have reflected the accumulation of a drive for energy replacement rather than the cycling of some internal oscillator. Means to distinguish between such possibilities (by altering meal times and/or the content of the food given) present ethical difficulties and have not been tested systematically. Nevertheless, in principle, if the drive for food intake were wholly due to energy replacement, then changing the energy value of the food eaten would produce a proportional change in the interval between feeds; but if the drive were due only to an oscillator then the timing of food intake would be unchanged, even though the amount taken would vary inversely with its energy content. The issue awaits resolution.

In adults, the gastrointestinal and metabolic effects produced by meals also show circadian changes (Jorde \& Burhol, 1985; Goo et al. 1987; Van Cauter et al. 1989,1991; Kumar, 1994; Cella et al. 1995; Wolever \& Bolognesi, 1996). Again this is mainly due to social customs. In aged subjects, who tend to be more regular than younger controls in their lifestyle (if only because they have less potentially-disturbing influences from the demands of occupation and child-rearing), meal times, size and composition, can vary hardly at all during the course of 1 week (Minors et al. 1989). Even so, there is some evidence to indicate that there exists, in addition to social influences, a weak circadian rhythm in the response to food intake; for example, rhythms in: the response to a glucose load (Sensi, 1974; Owens et al. 1997) which is less rapid in the evening than the morning; gastric $\mathrm{pH}$, 
Table 2. Summary of factors which might cause rhythmicity in food intake

\begin{tabular}{l} 
Internal \\
Circadian oscillator \\
Ultradian oscillator \\
Biochemical need for food \\
External factors \\
Environmental (light $v$. dark) \\
Behavioural (active $v$. asleep) \\
Opportunistic (meal break, cafeteria open) \\
Habitual (the 'correct' or customary clock time) \\
Social (communal meals, neonate with nursing mother) \\
\hline
\end{tabular}

both in the morning and after a meal (Tarquini et al. 1987; Barlow et al. 1994); the rate of gastric emptying and gut muscle activity (Goo et al. 1987; Kumar, 1994); and in the absorption of drugs and foodstuffs from the gastrointestinal tract due to changes in gastric emptying and blood flow (Sanders \& Moore, 1992), both of which are greater in the daytime than at night. This last finding has an important implication for constant-routine studies (see p. S30) if the metabolism of digested foodstuffs is important. Thus, ingestion of identical, equally-spaced meals throughout the $24 \mathrm{~h}$ does not result in a constant absorption of foodstuffs from the gut; subjects report feeling bloated during the night. A method of constant intravenous infusion of glucose (Van Cauter et al. 1991) corrects this (but does nothing to control circadian changes in sequestration or metabolism). A summary of the factors which might lead to rhythmicity in food intake is given in Table 2.

\section{MEAL TIMES AS ZEITGEBERS}

The possibility exists that meal times can act as a zeitgeber and help adjust individuals' circadian rhythms. There are three possibilities. First (see Reppert, 1989), since melatonin appears in breast milk, the mother might be able to influence her baby by this route. This does not seem to have been investigated and it assumes that the baby's circadian system has developed sufficiently for the SCN to respond to any melatonin. This might not be so in human subjects, although there is evidence for such responsiveness to melatonin in rodents. Moreover, in human subjects as in rodents, suckling fulfils a social role and, if performed regularly, could be a second way of acting as a time cue or zeitgeber.

A third mechanism has been suggested by which meals might act as a zeitgeber in adults and this is based on changes in the balance of amino acids in the plasma following protein-rich or carbohydrate-rich meals (summarized by Leathwood, 1989). Briefly, protein-rich meals raise plasma tyrosine in preference to plasma tryptophan; carbohydraterich meals have the opposite effect. The argument continues that this could raise the production of catecholamine and serotonin neurotransmitters respectively (since these amino acids are the precursors of these neurotransmitters). This would, in turn, promote the release of the transmitters causing alertness (catecholamines) or sleepiness (serotonin). Work with rodents has not supported the hypothesis (Leathwood, 1989). Thus, it has not been shown that precursor uptake is the rate-limiting step for catecholamine or serotonin release, or that such changes in release are responsible for changes in alertness. Such tests of this feeding hypothesis that have been conducted on human subjects have not been well 
designed and the hypothesis remains unproved in this species also. Even so, kits containing two types of pill (one with tyrosine, for morning ingestion, and the other with tryptophan, for evening ingestion) have appeared with claims that they promote adjustment of the body clock and so combat 'jet-lag'.

\section{FOOD INTAKE DURING NIGHT WORK}

Estimates are that approximately $20 \%$ of the workforce of industrialized societies is involved in night work at some stage. Epidemiological evidence indicates that gastric ulcers are more common in night workers than in dayworking colleagues, and that indigestion is common, together with irregularity of bowel movements (Waterhouse et al. 1992). The cause of these problems is multifactorial, involving changes in lifestyle and endogenous factors. The obvious change in lifestyle, the 'lunch break' is in the middle of the night!, might cause problems insofar as circadian rhythms in gut function exist (see p. S33). Other factors are probably the consumption of tobacco and caffeine (to help stay awake during the night) and alcohol (to help getting to sleep in the morning), all of which are increased in nightworkers (Rutenfranz et al. 1977).

A closely associated problem is that there is a loss of appetite and a feeling of being bloated. Studies show that even though the total quantity of food eaten during the $24 \mathrm{~h}$ is not necessarily changed, its timing with respect to work hours is altered; lunch is more of a snack than during day work. Indeed, there is a tendency for workers to 'graze' their way through night shifts rather than have a more substantial meal (Krauchi et al. 1990; Reinberg et al. 1979; Nikolova et al. 1990). In some cases this is because cafeteria facilities are not available at night, local take-away restaurants are closed or because the food that is available is not palatable; in others, it is because it is believed that night is the 'wrong' time to eat a full meal. Moreover, when at home, meals can become monotonous. 'Every meal seemed like breakfast', implying a preponderance of fried food, summarizes the experience of some night workers in the UK. This arises because the times of meals can be inconvenient to other members of the household (particularly if they have to prepare a meal for only one person, they have other tasks to perform or they would rather be asleep) and ease and speed of preparation can be a major factor. Clearly, the exact nature of the change in eating habits will depend on the culture, but some form of alteration is universal. It is also likely to be particularly marked during religious festivals, for example, the holy month of Ramadan (Khashoggi et al. 1993).

Clearly there is a challenge here to improve the general food hygiene of night workers, particularly with the advent of the microwave oven and the increasing awareness of a healthy diet, with more fibre and fresh produce and less fried food and cholesterol-rich foodstuffs. A recent study (Niedhammer et al. 1996) lends support to this argument, since nurses working night shifts were found to be more likely to be obese than their dayworking colleagues.

\section{WHAT WE NEED TO KNOW}

A detailed study of what and when we eat is needed. This could investigate the extent to which these variables depend on the social concomitants of a meal, our customs and the opportunity to eat, as well as how hungry we feel. Moreover, our appetite might be influenced by internal circadian and ultradian oscillators as well as these aspects of lifestyle. The problem is marked in night workers in whom appetite is poor, indigestion is 
common, and there is an increased incidence of gastrointestinal disorders in comparison with day workers. Therefore, the study could focus upon these individuals as well as day workers.

\section{Night workers}

(a) A large number of people are involved in nightwork for substantial portions of their lives and problems with appetite, indigestion etc. are common. A means to promote appropriate eating patterns during night work would be highly desirable.

(b) It is not known to what extent altered patterns of food intake reflect: (1) altered desire for food in general; (2) unpalatability of the kind of food that is available; (3) difficulties of food preparation and/or consumption; (4) effects due to circadian and/or ultradian oscillators.

(c) It is proposed that a systematic study of these factors in nightworkers be carried out; the same workers during day shifts or rest days could act as controls.

(d) The use of suitable questionnaires together with a suitable array of food types and convenient preparation facilities would enable some of these issues to be resolved.

(e) There would be very considerable implications of the findings. For example, is the need to provide better food preparation facilities, more palatable food or to educate the workforce to alter its pattern of eating during night work? Alternatively, is the main problem an unadjusted 'body clock', which implies the adoption of shift schedules, amenities in the work place and general lifestyles that promote adjustment to night work?

\section{REFERENCES}

Aschoff, J., von Goetz, C., Wildgruber, C. \& Wever, R. (1986). Meal timing in humans during isolation without time cues. Journal of Biological Rhythms 1, 151-162.

Barlow, A., Hinder, R. \& DeMeester, T. (1994). Twenty-four-hour gastric luminal pH in normal subjects: influence of probe position, food, posture, and duodenal reflux. American Journal of Hypertension 90, 11861187.

Bernstein, I., Zimmerman, J., Czeisler, C. \& Weitzman, E. (1981). Meal patterns in 'free-running' humans. Physiology and Behavior 27, 621-623.

Cella, L., Van Cauter, E. \& Schveller, D. (1995). Effect of meal timing in diurnal rhythm of human cholesterol synthesis. American Journal of Physiology 269, E878-E883.

Czeisler, C., Richardson, G., Zimmerman, J., Moore-Ede, M. \& Weitzman, E. (1981). Entrainment of human circadian rhythms by light-dark cycles: a reassessment. Photochemistry and Photobiology 34, $239-247$.

Dijk, D.-J. \& Czeisler, C. (1995). Contribution of the circadian pacemaker and the sleep homeostat to sleep propensity, sleep structure, and electroencephalographic slow waves, and spindle activity in humans. Journal of Neuroscience 15, 3526-3538.

Editorial (1984). Pulsatile control of reproduction. Lancet ii, 382-383.

Goo, R., Moore, J., Greenberg, E. \& Alazraki, N. (1987). Circadian variation in gastric emptying of meals in humans. Gastroenterology 93, 515-518.

Hellbrugge, T. (1973). Ultradian rhythms in childhood. International Journal of Chronobiology 1, 331-348.

Honnebier, M., Swaab, D. \& Mirmiran, M. (1989). Diurnal rhythmicity during early human development. In Development of Circadian Rhythmicity and Photoperiodism in Mammals, pp. 221-244 [S. Reppert, editor]. New York: Perinatology Press.

Jorde, R. \& Burhol, P. (1985). Review. Diurnal profiles of gastrointestinal regulatory peptides. Scandinavian Journal of Gastroenterology 20, 1-4.

Jorde, R., Jenssen, T. \& Burhol, P. (1987). Cyclic patterns of plasma pancreatic polypeptide (PP), plasma motilin, serum insulin and plasma gastric inhibitory polypeptide (GIP) in fasting man. Italian Journal of Gastroenterology 19, 10-12.

Khashoggi, R., Madani, K., Ghaznawi, H. \& Ali, M. (1993). The effects of Ramadan fasting on body weight. Joumal of Islamic Medical Association of North America 25, 44-45.

Kleitman, N. (1982). Basic rest-activity cycle -22 years later. Sleep 5, 311-317.

Krauchi, K., Nussbaum, P. \& Wirz-Justice, A. (1990). Consumption of sweets and caffeine in the night shift: relation to fatigue. In Sleep '90, pp. 62-64 [J. Horne, editor]. Bochum: Pontenagel Press. 
Kumar, D. (1994). Sleep as a modulator of human gastrointestinal motility. Gastroenterology 107, 1548-1550.

Lavie, P. (1980). Ultradian rhythmicity (circa $90 \mathrm{~min}$ ) in physiologic processes - evidence for multioscillatory phenomena. Journal of Interdisciplinary Cycle Research 11, 171-177.

Lavie, P. \& Kripke, D. (1981). Mini review. Ultradian circa 1.5 hour rhythms: a multioscillatory system. Life Science 29, 2445-2450.

Leathwood, P. (1989). Circadian rhythms of plasma amino acids, brain neurotransmitters and behaviour. In Biological Rhythms in Clinical Practice, pp. 136-159 [J. Arendt, D. Minors and J. Waterhouse, editors]. London: John Wright.

Lewy, A., Ahmed, S., Jackson, J. \& Sack, R. (1992). Melatonin shifts circadian rhythms according to a phaseresponse curve. Chronobiology International 9, 380-392.

Lloyd, D. \& Kippert, F. (1993). Intracellular co-ordination by the ultradian clock. Cell Biology International Reports 17, 1047-1052.

Maeda, K.-I., Tsukamura, H., Ohkura, S., Kawakami, S., Nagabukuro, H. \& Yokoyama, A. (1995). The LHRH pulse generator: a mediobasal hypothalamic location. Neuroscience and Behavioral Reviews 19, 427-437.

Mejean, L., Bicakova-Rocher, A., Kolopp, M., Villanme, C., Levi, F., Debry, G., Reinberg, A. \& Drouin, P. (1988). Circadian and ultradian rhythms in blood glucose and plasma insulin of healthy adults. Chronobiology International 5, 227-236.

Minors, D., Rabbitt, P., Worthington, H. \& Waterhouse, J. (1989). Variation in meals and sleep-activity patterns in aged subjects: its relevance to circadian rhythm studies. Chronobiology International 6, 139-146.

Minors, D. \& Waterhouse, J. (1981). Circadian Rhythms and the Human. Bristol: John Wright.

Moore, R. (1992). The organisation of the human timing system. Progress in Brain Research 93, 101-117.

Moore-Ede, M. (1986). Physiology of the circadian timing system: predictive versus reactive homeostasis. American Journal of Physiology 250, R737-R752.

Moore-Ede, M., Fuller, F. \& Sulzman, C. (1982). The Clocks that Time Us. Cambridge, MA: Harvard University.

Niedhammer, I., Lert, F. \& Marne, M. (1996). Prevalence of overweight and weight gain in relation to night work in a nurses cohort. International Journal of Obesity 20, 625-633.

Nikolova, N., Handjiev, S. \& Angelova, K. (1990). Nutrition of night and shiftworkers in transports. In Shiftwork: Health, Sleep and Performance, pp. 538-547 [G. Costa, G. Cesana, K. Kogi and A. Wedderburn, editors]. Frankfurt: Peter Lang.

Owens, D., Macdonald, I., Benton, D., Sytnik, N., Tucker, P. \& Folkard, S. (1997). A preliminary investigation into individual differences in the circadian variation of meal tolerance: effects on mood and hunger. Chronobiology International (In the Press).

Reilly, T. (1990). Human circadian rhythms and exercise. Critical Reviews in Biomedical Engineering 18, 165180.

Reinberg, A., Migraine, C. \& Apfelbaum, M. (1979). Circadian and ultradian rhythms in the eating behaviour and nutrient intake of oil refinery operators (Study 2). Chronobiologia Suppl. 1, 89-102.

Reppert, S. (editor) (1989). Development of Circadian Rhythmicity and Photoperiodism in Mammals. New York: Perinatology Press.

Reppert, S., Weaver, D., Rivkees, S. \& Stupa, E. (1988). Putative melatonin receptors in a human biological clock. Science 242, 78-81.

Rutenfranz, J., Colquhoun, P. \& Knauth, P. (1977). Biomedical and psychosocial aspects of shift work. A review. Scandinavian Journal of Work Environment and Health 3, 165-182.

Sanders, S. \& Moore, J. (1992). Gastrointestinal chronopharmacology: physiology, pharmacology and therapeutic implications. Pharmacology and Therapeutics 54, 1-15.

Sensi, S. (1974). Some aspects of circadian variations of carbohydrate metabolism and related hormones in man. Chronobiologia 1, 396-404.

Stagner, J., Samols, E. \& Weir, G. (1980). Sustained oscillations of insulin, glucagon, and somatostatin from the isolated canine pancreas during exposure to a constant glucose concentration. Journal of Clinical Investigation 65, 939-942.

Tarquini, B., Lombardi, P., Pernice, L., Cariddi, A., Checchi, M., Masi, L. \& Andreoli, F. (1987). Intragastric pH continuously monitored in health and in gastric diseases: chronobiologic assessment. In Advances in Chronobialogy A, pp. 379-387 [E. Haus, F. Halberg and W. Rietveld, editors]. New York: Arthur Liss Associates.

Van Cauter, E., Blackman, J., Roland, D., Spire, J.-D., Refetoff, S. \& Polonsky, K. (1991). Modulation of glucose regulation and insulin secretion by circadian rhythmicity and sleep. Journal of Clinical Investigation 88, 934-942.

Van Cauter, E., Desir, D., Decoster, C., Fery, F. \& Balasse, E. (1989). Nocturnal decrease in glucose tolerance during constant glucose infusion. Journal of Clinical Endocrinology and Metabolism 69, 604-611.

Veldhuis, J. (1994). Pulsatile hormone release as a window into the brain's control of the anterior pituitary gland in health and disease: implications and consequences of pulsatile luteinizing hormone secretion. Endocrinologist 4, 454-469. 
Waterhouse, J., Folkard, S. \& Minors, D. (1992). Shiftwork, Health and Safety. An Overview of the Scientific Literature 1978-1990. London: H. M. Stationery Office.

Weinert, D., Sitka, U., Minors, D. \& Waterhouse, J. (1994). The development of circadian rhythmicity in neonates. Early Human Development 36, 117-126.

Wever, R. (1979). The Circadian System of Man. Berlin: Springer Verlag.

Wolever, T. \& Bolognesi, C. (1996). Time of day influences relative glycaemic effect of foods. Nutrition Research 16, 381-384. 